\title{
Efficacy and safety of multiple doses of tapentadol oral solution in the treatment of moderate to severe acute pain in children aged 2 to $<18$ years - a randomized, double-blind, placebo-controlled trial
}

This article was published in the following Dove Press journal:

Journal of Pain Research

\author{
Christoph Beuter (ID) \\ Gisela Volkers (1D) \\ Tatjana Radic' \\ Jutta Goldberg' \\ John van den Anker ${ }^{2,3}$ \\ 'Grünenthal GmbH, Aachen, Germany; \\ ${ }^{2}$ Division of Paediatric Pharmacology and \\ Pharmacometrics, University of Basel \\ Children's Hospital, Basel, Switzerland; \\ ${ }^{3}$ Division of Clinical Pharmacology, \\ Children's National Medical Center, \\ Washington, DC, USA
}

\begin{abstract}
Background: Well-controlled trials of analgesics in the pediatric population are scarce. Tapentadol is a strong centrally acting analgesic which has undergone a pediatric development program investigating its suitability for treating moderate to severe acute pain across the entire pediatric age range from birth to adolescence. Here, we report data from a pivotal Phase III trial performed as part of this development program.

Patients and methods: This randomized, double-blind, placebo-controlled, multicenter clinical trial investigated efficacy and safety/tolerability of multiple tapentadol oral solution doses (OS; target dose $1.25 \mathrm{mg} / \mathrm{kg}$ ) in the treatment of postsurgical acute pain. Data for patients aged 2 to $<18$ years are reported here. The main objective of the trial was to investigate if oral tapentadol administration compared to placebo reduces the use of supplemental opioid analgesic medication within the first $24 \mathrm{hrs}$ of treatment. Other investigated parameters included taste and palatability of the trial medication, adverse events (AEs), vital signs, and laboratory parameters.
\end{abstract}

Results: A total of 160 patients were included (placebo $n=52$, tapentadol $n=108$ ). It was shown that the total amount of supplemental opioid analgesic medication used in the first $24 \mathrm{hrs}$ was significantly lower in tapentadol patients than placebo patients $(\mathrm{p}=0.0154)$. Taste and palatability of tapentadol OS was well perceived by most patients. Treatment-emergent AEs were reported in $50 \%$ of patients treated with placebo vs $57.4 \%$ in those exposed to tapentadol, most commonly vomiting, nausea, and constipation in both treatment groups.

Conclusion: Tapentadol OS was effective and generally well tolerated in children ( $\geq 2$ years) for the treatment of moderate to severe acute pain. Across all age groups, palatability and acceptability of tapentadol OS were sufficient to ensure intake compliance. This trial provides evidence that tapentadol OS can be effectively used to treat pain in young patients for whom currently limited labelled treatment options are available.

Keywords: tapentadol oral solution, pediatric pain management, moderate to severe acute pain, efficacy, safety

\section{Introduction}

In the past, systematic clinical trial programs in the pediatric population for drugs initially targeted for the adult population were often not carried out. As a result, off-label use of medicines in the pediatric population has become common practice. ${ }^{1}$ This lack of systematic evaluation of medicinal products has led to a regulatory framework for the study of new drugs in the pediatric population
Correspondence: Christoph Beuter Grünenthal GmbH, Zieglerstraße 6, 52078 Aachen, Germany

Tel +4924l 5690

Email Christoph.Beuter@grunenthal.com 
initiated by authorities such as the European Medicines Agency $^{2}$ and the United States Food and Drug Administration. ${ }^{3}$ However, current treatment recommendations for the pediatric population are often still based on empirical data and expert opinions. For instance, guidelines for pediatric pain management after surgery recommend a multimodal analgesia approach depending on the severity of pain experienced by the child or adolescent. ${ }^{4,5}$ Following these guidelines, opioids are broadly administered for the treatment of moderate to severe acute pediatric pain ${ }^{4,5}$ but often have not been tested in clinical trials in this indication and are thus used in an off-label manner.

Tapentadol, a strong centrally acting analgesic with a dual mechanism of action ${ }^{6}$ has been shown to provide effective pain relief across multiple indications in the adult population. $^{7-15}$ It has a beneficial gastrointestinal tolerability with lower rates of nausea, vomiting, and constipation compared to commonly used opioids. With its dual mechanism of action, including noradrenaline reuptake inhibition (NRI) in addition to $\mu$-opioid receptor agonism (MOR), tapentadol provides equianalgesia with a lower burden of typical $\mu$-opioid receptor-related side effects in comparison to pure opioids due to an overall lower $\mu$-load. ${ }^{16}$ The $\mu$-load describes the contribution (\%) of the MOR component to the adverse effect magnitude relative to a pure/classical $\mu$-opioid at equianalgesia and has been estimated at $\leq 40 \%$ for tapentadol. ${ }^{16}$ Forty percent or less of the overall effect is due to MOR agonism, the remainder is resulting from NRI reuptake inhibition and the synergy of the two mechanisms of action. Morphine milligram equivalents to achieve equianalgesia are based on a conversion factor of 2.5:1 (tapentadol:morphine). With this conversion, comparable analgesic effects of tapentadol and morphine are reached, however, because of the lower $\mu$-load of $\leq 40 \%$, not the same opioid activity will be reached. In addition to its favourable side effect profile, tapentadol has a predictable pharmacokinetic (PK) profile with no active metabolites ${ }^{6}$ and a low potential for PK drug-drug interactions. ${ }^{17,18}$ Based on this, tapentadol was identified as a potentially well suited treatment alternative for moderate to severe pain in the pediatric population. It underwent a multi-national pediatric development program for the entire age range from preterm neonates to adolescents $<18$ years in agreement with EU and US authorities.
As part of this program, a randomized, double-blind, placebo-controlled multicenter Phase III trial was performed in order to evaluate efficacy and safety/tolerability across the age range from birth to $<18$ years. Data of patients aged 2 years and older are reported here. Data for patients $<2$ years will be presented in a later publication. An oral solution (OS) was identified as the appropriate formulation to dose patients in this trial. Oral tapentadol solutions were already available at the start of the pediatric program: a $20 \mathrm{mg} / \mathrm{mL}$ solution was approved and marketed in several countries for adult use and a novel $4 \mathrm{mg} / \mathrm{mL}$ had been developed in preparation of the pediatric development program. In order to identify a tapentadol dose for the population reported here, a pediatric population PK model was built using data from two dedicated single dose PK trials. ${ }^{19,20}$ Simulations conducted using this model indicated a tapentadol dose of $1.25 \mathrm{mg} / \mathrm{kg}$ body weight given every $4 \mathrm{hrs}$ as appropriate. ${ }^{21}$ This dose was expected to produce exposures (AUC $\tau, \mathrm{ss}$ ) at steady state within the targeted range in adults achieved after repeat dosing of 50-100 mg tapentadol immediate-release every 4 hrs; these doses have previously been shown to be safe and efficacious in adults. ${ }^{7-14}$ Full details of dose selection are provided in a parallel pharmacometric publication in this thematic tapentadol series. ${ }^{21}$

\section{Patients and methods Trial design}

This randomized, double-blind, placebo-controlled, parallel group, multicenter Phase III trial was conducted in accordance with the Declaration of Helsinki, Good Clinical Practice, national regulations, and applicable local laws at 44 trial sites located in 9 European countries and the United States (supplementary document). The trial period for the group of children and adolescents from 2 to $<18$ years of age was from February 2015 to December 2016. Trial protocol and amendments, patient information sheet, and informed consent/assent forms were approved by independent ethics committees or institutional review boards at the participating trial sites. An assent or a written informed consent was obtained from the participating patients, as applicable. In addition, their parents/legal guardians provided written informed consent as legally required. The trial is registered with Eudra-CT (No. 2012-004359-35).

Figure 1 summarizes the trial design. The trial consisted of an enrollment period (up to 28 days before allocation to trial medication), a double-blind treatment 


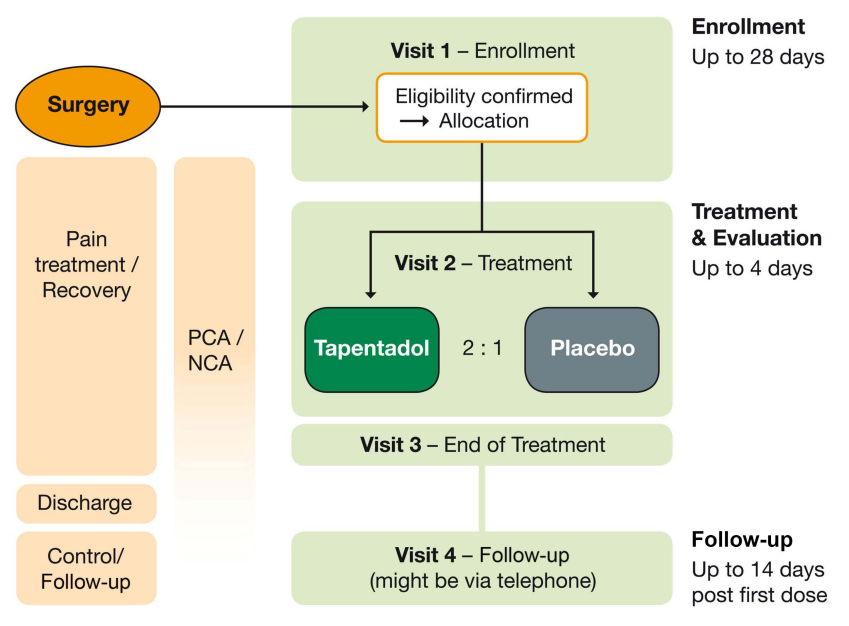

Figure I Trial design.

Abbreviations: NCA, nurse-controlled analgesia; PCA, patient-controlled analgesia.

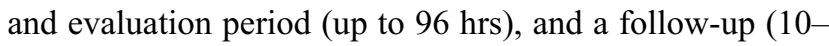
14 days after first dose of trial medication; might be performed by telephone). Patients were enrolled before or after surgery. During recovery from surgery, they received postsurgical standard of care treatment via patient-controlled analgesia (PCA) or nurse-controlled analgesia (NCA) with morphine or hydromorphone (with or without a background opioid infusion), eligibility for trial participation was confirmed, and patients were then allocated to treatment.

Employing a standard double-blind trial design to determine the efficacy of an analgesic medication by showing superiority over placebo based on pain assessments might leave patients in the placebo arm without sufficient pain relief and is not ethically acceptable for pediatric trials. ${ }^{1}$ Therefore, an immediate rescue design with an alternative efficacy endpoint was chosen. This endpoint allowed to compare the use of supplemental opioid analgesic medication via PCA or NCA between study participants receiving placebo or active trial medication. Opioids permitted were morphine and hydromorphone. Using this approach, efficacy could be determined, and all pediatric patients still had access to adequate pain treatment. This approach has been recommended for pain trials in children ${ }^{22,23}$ and has been used previously. ${ }^{24}$ The dose of tapentadol OS was $1.25 \mathrm{mg} / \mathrm{kg}$ every $4 \mathrm{hrs}$.

\section{Patients}

Male and female patients were eligible to participate if they had undergone surgery that, in the investigator's opinion, would lead to moderate to severe acute pain that would require opioid treatment for at least $24 \mathrm{hrs}$ after the first dose of trial medication and were hospitalized until the end of treatment. Brain surgeries increasing the risk of CNS side effects or gastrointestinal surgery procedures expected to affect tapentadol absorption were not included. Patients should have received morphine or hydromorphone as PCA or NCA with or without a background infusion of the same opioid (i.e., morphine or hydromorphone) according to standard of care prior to allocation to trial medication. Patients were expected to continue requiring opioid therapy by NCA/PCA after start of trial medication intake. Main exclusion criteria were concomitant diseases or disorders that, in the opinion of the investigator, could affect or compromise patient safety during the trial, a current condition or history of CNS disorders or diseases (such as non-febrile seizure disorder, epilepsy, serotonin syndrome, traumatic or hypoxic brain injury, brain tumor), moderate to severe renal or hepatic impairment as confirmed by local screening laboratory results, abnormal pulmonary function or clinically relevant respiratory disease, postsurgical clinically relevant abnormal laboratory or hematology values, or clinically relevant abnormal electrocardiogram (ECG) findings. Subjects had to be clinically stable postsurgically and mentally capable of complying with the trial procedures. The following also led to exclusion: previous exposure to tapentadol; clinically relevant history of hypersensitivity, allergy, or contraindication to the supplemental opioid analgesic medication, tapentadol, the excipients, or naloxone; history of suicidal ideation or behavior; history of alcohol and/or substance abuse; or breastfeeding.

The following medications/procedures were prohibited for prespecified time frames prior to and following trial medication allocation: monoamine oxidase inhibitors, strong enzyme inducing drugs, methadone, neuroleptics, anticonvulsants (except for gabapentin used in association with surgery), antiparkinsonian drugs, all serotonergic drugs including selective serotonin/norepinephrine reuptake inhibitors, tricyclic antidepressants, linezolid, triptans, St. John's Wort, long-acting opioids, sedatives (except for benzodiazepines for muscle spasms or anxiety), or peri- or postsurgical analgesia supplied by a continuous regional technique or patient-controlled epidural analgesia, opioid analgesics other than morphine, hydromorphone, and tapentadol, as well as continuous positive airway pressure or mechanical ventilation.

\section{Randomization}

Eligible patients were randomly allocated (2:1) to either tapentadol OS or a matching placebo OS using an 
interactive voice/web response system. Randomization for the trial population from 2 to $<18$ years followed computer-generated randomization lists balanced by permuted blocks and stratified by age groups and supplemental opioid use (morphine or hydromorphone). Patients, investigators, and other trial team members involved in trial conduct, data management, or trial analysis remained blinded to trial medication until after data lock.

\section{Treatment}

Patients received tapentadol OS doses of $1.25 \mathrm{mg} / \mathrm{kg}$ or the corresponding volume of placebo OS for the first $24 \mathrm{hrs}$ at dosing intervals of $4 \mathrm{hrs}$ ( $\pm 15 \mathrm{mins})$. The maximum permitted individual dose was $100 \mathrm{mg}$ tapentadol per administration. If asleep at the time of the scheduled dose, the patient was awakened and dosed within a maximum time of $6 \mathrm{hrs}$ after the previous dose. Background infusions were discontinued at administration of first trial medication. If a patient had unbearable pain despite using PCA/ $\mathrm{NCA}$, an additional bolus (defined as a clinician bolus) of morphine or hydromorphone (whichever opioid was administered by PCA/NCA) could be given either using the PCA/NCA pump system or by an intravenous bolus injection. If, in the investigator's opinion, analgesic requirements had declined after $24 \mathrm{hrs}$, tapentadol doses could be reduced to $1 \mathrm{mg} / \mathrm{kg}$. Dosing was stopped at the latest after $72 \mathrm{hrs}$ of treatment, or if the patient was switched to exclusively oral opioid analgesics, or if opioid analgesics were no longer required.

Vital signs, sedation score, oxygen saturation, and pain scores were measured before each administration of trial medication.

\section{Efficacy assessments}

Table 1 lists the efficacy outcome measures. The primary efficacy endpoint assessed the difference between the treatment arms in the "total amount of supplemental opioid analgesic medication used within the first $24 \mathrm{~h}$ after first dose of trial medication" expressed as morphine equivalents in $\mathrm{mg} / \mathrm{kg}$ body weight (to obtain the morphine equivalent, hydromorphone doses were multiplied by 5). Supplemental opioid analgesic medications included in the analysis were opioids given via PCA/ NCA, clinician bolus, and other intravenously administered opioids. Secondary efficacy endpoints included the total amount of supplemental opioid analgesic medication used within the first $12 \mathrm{hrs}$ after first dose, the total amount of supplemental opioid analgesic medication
Table I Efficacy Outcome Measures

\begin{tabular}{|c|c|}
\hline Outcome measure & Description \\
\hline $\begin{array}{l}\text { Amount of supplemental } \\
\text { opioid analgesic medication }\end{array}$ & $\begin{array}{l}\text { Morphine or hydromorphone } \\
\text { administered via PCA/NCA }\end{array}$ \\
\hline \multicolumn{2}{|l|}{$\begin{array}{l}\text { Pain intensity, assessed using } \\
\text { age-appropriate rating scales }\end{array}$} \\
\hline $\begin{array}{l}\text { For patients } 2 \text { to }<6 \text { years or } \\
\text { older children incapable of } \\
\text { communicating their pain: } \\
\text { Face, Legs, Activity, Cry, and } \\
\text { Consolability }\left(\mathrm{FLACC}^{25}\right) \text { scale }\end{array}$ & $\begin{array}{l}\text { Observational scale from } 0 \text { to } 10 \\
\text { with } 0=\text { no pain }\end{array}$ \\
\hline $\begin{array}{l}\text { For patients } 6 \text { to }<12 \text { years: } \\
\text { Faces Pain Scale-revised } \\
\left(\text { FPS- }{ }^{26}\right)\end{array}$ & $\begin{array}{l}\text { Self-reporting } 6 \text {-point scale } \\
\text { Six faces with scores of } 0,2,4,6,8 \text {, } \\
10 \text { where } 0=\text { no pain and } 10=\text { very } \\
\text { much pain }\end{array}$ \\
\hline $\begin{array}{l}\text { For patients } 12 \text { to }<18 \text { years: } \\
\text { Visual Analog Scale }\end{array}$ & $\begin{array}{l}\text { Self-reporting } 100 \mathrm{~mm} \text { scale from } \\
0=\text { no pain to } 100=\text { worst imaginable } \\
\text { pain }\end{array}$ \\
\hline $\begin{array}{l}\text { Clinical Global Impression of } \\
\text { Change }\left(\mathrm{CGIC}^{27}\right)\end{array}$ & $\begin{array}{l}\text { Investigator rating of patients' } \\
\text { overall improvement from I=very } \\
\text { much improved to } 7=\text { =very much } \\
\text { worse }\end{array}$ \\
\hline $\begin{array}{l}\text { Patient Global Impression of } \\
\text { Change }\left(\mathrm{PGIC}^{27}\right)\end{array}$ & $\begin{array}{l}\text { Patients or parents/guardians rating } \\
\text { of overall change in patients' health } \\
\text { status from I=very much improved } \\
\text { to } 7=\text { very much worse }\end{array}$ \\
\hline $\begin{array}{l}\text { Palatability and acceptability of } \\
\text { trial medication }\end{array}$ & $\begin{array}{l}\text { 5-point faces scale }{ }^{28} \text { from really } \\
\text { good/easy to really bad/really } \\
\text { difficult }\end{array}$ \\
\hline
\end{tabular}

Abbreviations: NCA, nurse-controlled analgesia; PCA, patient-controlled analgesia.

received from 24 to $96 \mathrm{hrs}$ after first dose assessed in 12-hr intervals, changes from baseline in pain intensity over the treatment period, palatability and acceptability after first and last dose of trial medication, Clinical Global Impression of Change (CGIC) and Patient Global Impression of Change (PGIC) ratings after completion of double-blind treatment, and time to treatment discontinuation due to lack of efficacy.

\section{Safety and tolerability assessments}

Adverse events (AEs) were monitored throughout the trial. Tolerability was assessed by analyzing all treatment-emergent adverse events (TEAEs; any AEs that occurred at or after first administration of placebo or tapentadol). The proportion of patients discontinuing treatment due to TEAEs or drug-related TEAEs was also analyzed. 
Safety assessments additionally included physical examination, clinical chemistry and hematology, 12-lead ECG, vital signs, oxygen saturation by pulse oximetry, and University of Michigan Sedation Scale scores. Suicidal ideation/behavior was assessed in patients $\geq 6$ years before first trial medication administration and at the end of treatment using the Columbia-Suicide Severity Rating Scale. $^{29}$

\section{Statistical analysis}

Sample size calculations were based on published singledose trials in postsurgical pediatric subjects where supplemental opioid was measured. ${ }^{30,31}$ The treatment difference between tapentadol and placebo was assumed to be 0.2 $\mathrm{mg} / \mathrm{kg}$ (SD $0.42 \mathrm{mg} / \mathrm{kg}$ ) of supplemental opioid requirement within the first $24 \mathrm{hrs}$ after first dose. Assuming a two-sided significance level of $5 \%$, a power of $80 \%$ and a randomization ratio of 2:1 (tapentadol to placebo) resulted in a sample size of 106 patients in the tapentadol group and 53 patients in the placebo group.

All analyses were performed with SAS version 9.3 or later (SAS Institute Inc., Cary, USA). All randomized patients who received at least one dose of trial medication were analyzed for safety (safety set $[\mathrm{SAF}]$ ) and efficacy (full analysis set, FAS).

The primary null hypothesis to be rejected was that the tapentadol group would not be different from the placebo group for the primary efficacy endpoint, i.e., children treated with tapentadol would need the same amount of supplemental opioid as those treated with placebo. The alternative hypothesis was that children treated with tapentadol would need less supplemental opioid compared to placebo. The groups were compared for the primary endpoint using an analysis of variance (ANOVA) model, with treatment, baseline age group, and the supplemental opioid analgesic used (morphine vs hydromorphone) as factors. The test for the primary efficacy analysis was 2-sided at a 0.05 level of significance. For patients discontinuing treatment earlier than $24 \mathrm{hrs}$ (because opioid analgesic medication was no longer needed or because they were switched to exclusively oral opioid analgesic medication), the observed supplemental opioid analgesic medication use until their timepoint of discontinuation was used for analysis. For patients discontinuing treatment due to other reasons, supplemental opioid analgesic medication usage was linearly imputed from their time of discontinuation until $24 \mathrm{hrs}$. To confirm the results, three sensitivity analyses were performed, one using the per-protocol population which included all FAS patients without major protocol deviations affecting the primary efficacy parameter, and two multiple imputation methods for missing data, placebo mean imputation and treatment mean imputation. An analysis of the primary endpoint was also performed including the amount of opioid analgesia taken prior to trial medication intake as a covariate into the primary ANOVA model.

The treatment comparison for the secondary efficacy endpoint "total amount of supplemental opioid analgesic medication used within the first $12 \mathrm{~h}$ after first dose" used the same ANOVA model employed for the primary endpoint. All other secondary endpoints were analyzed descriptively. CGIC and PGIC responders were defined as patients with at least "much improved" ratings at the end of treatment.

AEs were encoded using the Medical Dictionary for Regulatory Activities (MedDRA version 19.1). All TEAEs were sorted by MedDRA System Organ Class and Preferred Term and analyzed by age group ( 2 to $<6$ years, 6 to $<12$ years, 12 to $<18$ years) and overall for the trial population.

\section{Results \\ Patients}

A total of 165 patients were randomized to treatment (Figure 2). Five patients did not receive any trial medication, the safety and FAS set thus consisted of 160 patients (placebo $n=52$, tapentadol $n=108)$. More than half $(91 / 160$ [56.9\%]) completed $24 \mathrm{hrs}$ of treatment (placebo 28/52 [53.9\%], tapentadol 63/108 [58.3\%]). These patients all attended follow-up and completed the trial. Reasons for discontinuation are shown in Figure 2. Five of 52 placebo patients (9.6\%) and 13 of 108 tapentadol patients (12\%) discontinued early because opioid analgesic medication was no longer required, and 6/52 placebo patients (11.5\%) and 14/108 tapentadol patients (13\%) discontinued early because a switch to exclusively oral opioid analgesic medication was indicated according to local standard of care. Forty-six placebo and 94 tapentadol patients were included in the per-protocol set.

The trial population was mostly white (131/160 [81.9\%]); the proportion of male and female patients and patients in the three age groups ( 2 to $<6$ years, 6 to $<12$ years, 12 to $<18$ years) were well matched between the treatment arms (Table 2). Most patients (146/160 [91.3\%]) had concomitant diseases, mainly congenital, familial and genetic disorders (60/160 [37.5\%]), musculoskeletal and 


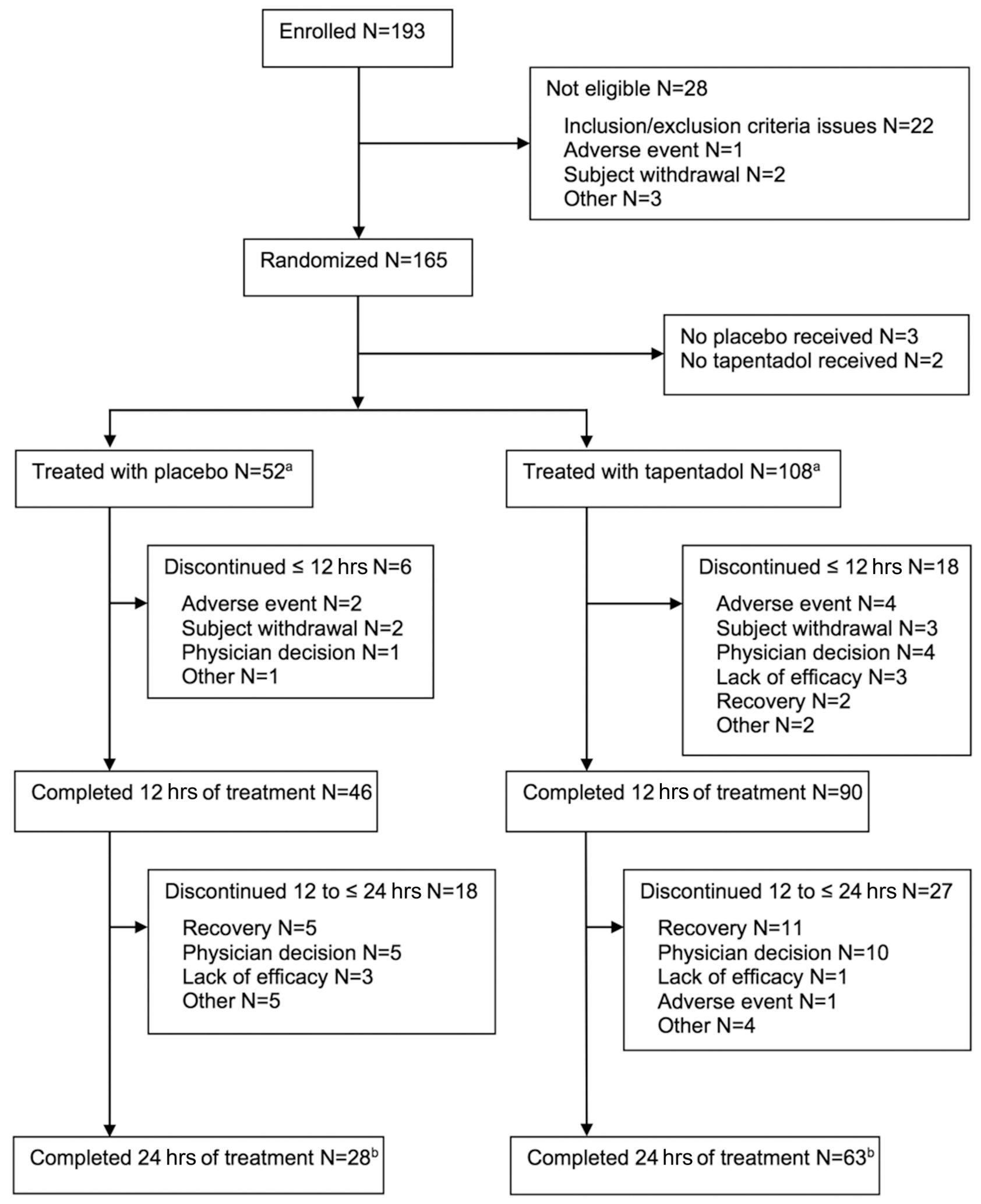

Figure 2 Patient flow chart.

Notes: "Recovery" denotes that opioid analgesic medication is no longer required. "Physician decision" denotes the switch to exclusively oral opioid analgesic medication indicated according to local standard of care. ${ }^{a}$ All patients treated with study medication were included in the full analysis set and the safety set. ${ }^{\mathrm{b}} \mathrm{All}$ patients attended the follow-up visit and completed the trial. 
Table 2 Demographic Data And Baseline Characteristics

\begin{tabular}{|c|c|c|c|}
\hline & $\begin{array}{l}\text { Placebo } \\
N=52\end{array}$ & $\begin{array}{l}\text { Tapentadol } \\
N=108\end{array}$ & $\begin{array}{l}\text { Overall } \\
N=160\end{array}$ \\
\hline \multicolumn{4}{|l|}{ Sex } \\
\hline Female & $23(44.2 \%)$ & 53 (49.1\%) & 76 (47.5\%) \\
\hline Male & $29(55.8 \%)$ & 55 (50.9\%) & $84(52.5 \%)$ \\
\hline \multicolumn{4}{|l|}{ Age group } \\
\hline 2 to $<6$ years & $12(23.1 \%)$ & 23 (21.3\%) & 35 (21.9\%) \\
\hline 6 to $<12$ years & 15 (28.8\%) & 32 (29.6\%) & 47 (29.4\%) \\
\hline 12 to $<18$ years & $25(48.1 \%)$ & 53 (49.1\%) & $78(48.8 \%)$ \\
\hline $\begin{array}{l}\text { Body mass index } \\
\left(\mathrm{kg} / \mathrm{m}^{2}\right)\end{array}$ & $19.1 \pm 3.8$ & $18.8 \pm 4.1$ & $18.9 \pm 4.0$ \\
\hline $\begin{array}{l}\text { Duration of } \\
\text { surgery (min) }\end{array}$ & $203.9 \pm 155.8$ & $186.0 \pm 110.5$ & $191.9 \pm 126.8$ \\
\hline $\begin{array}{l}\text { Background } \\
\text { infusion }^{\text {a }}\end{array}$ & 15 (28.8\%) & 39 (36.1\%) & $54(33.8 \%)$ \\
\hline $\begin{array}{l}\text { Intake of morphine } \\
\text { or hydromorphone } \\
\text { in the } 24 \text { hrs prior } \\
\text { to trial medication } \\
\left(\mathrm{mg} / \mathrm{kg}^{\mathrm{b}}\right)\end{array}$ & $0.45 \pm 0.7$ & $0.59 \pm 1.2$ & $0.55 \pm 1.1$ \\
\hline $\begin{array}{l}\text { Time between end } \\
\text { of surgery and first } \\
\text { dose of trial } \\
\text { medication ( } \mathrm{min})\end{array}$ & $795.9 \pm 553$ & $10 \mid 8.9 \pm 1483.8$ & $946.5 \pm|26| .3$ \\
\hline
\end{tabular}

Notes: Data are mean \pm standard deviation or number of patients (\%). ${ }^{2}$ Morphine or hydromorphone; ${ }^{\mathrm{b}}$ morphine equivalents.

connective tissue disorders (38/160 [23.8\%]), and gastrointestinal disorders $(20 / 160$ [12.5\%]). These diseases include the medical indication for the surgery which was the prerequisite for this trial. Surgeries affected all body systems with the exception of the brain. Distribution of surgery types was comparable between the treatment arms. Within a wide variety of surgery types, the most common were spinal fusion surgery $(22 / 160[13.8 \%])$, urethral repair (14/160 [8.8\%]), maxillofacial surgery (12/160 [7.5\%]), and thoracic surgery $(10 / 160[6.3 \%])$. Both treatment groups received comparable amounts of morphine or hydromorphone in the $24 \mathrm{hrs}$ prior to first dose of trial medication (Table 2). The most commonly used concomitant medications were analgesics including supplemental opioid analgesic medication (153/160 [95.6\%]), antibacterial agents for systemic use (115/160 [71.9\%]), blood replacements and perfusion solutions (111/160 [69.4\%]), anti-inflammatory and antirheumatic products $(67 / 160$ [41.9\%]), drugs for constipation (61/160 [38.1\%]), all of which are commonly used in a postsurgical setting. Overall 29.4\% (47/160) of the patients took medications to treat emesis or nausea. There was no change in the relative proportion of patients using these medications prior or concomitantly in both treatment groups. Although drugs for constipation were taken more often prior to tapentadol OS, the concomitant use of these medications was similar with placebo and tapentadol OS treatment.

\section{Treatment}

Mean exposure to trial medication was comparable between the groups with $28.3 \mathrm{hrs}$ (SD 17.3) for placebo and $28.9 \mathrm{hrs}$ (SD 18.0) for tapentadol patients. Most patients (placebo 43/52 [82.7\%], tapentadol 86/108 [79.6\%]) received at least five doses of trial medication within the first $24 \mathrm{hrs}$.

\section{Efficacy}

Supplemental opioid analgesic medication was more often controlled by the patient than by the nurse $(97 / 160$ [60.6\%] vs 59/160 [36.9\%], 4/160 [2.5\%] missing) which reflects the age distribution of the trial population and the distribution between self-reported and observed pain values. Morphine was more commonly used than hydromorphone (113/160 [70.6\%] vs 46/160 [28.8\%], 1/160 [0.6\%] missing). The most commonly used nonopioid within $24 \mathrm{hrs}$ after first trial medication was paracetamol as intravenous (17/52 [32.7\%] placebo, 24/108 [22.2\%] tapentadol patients) or oral formulation $(12 / 52$ [23.1\%] and $19 / 108[17.6 \%])$.

\section{Primary endpoint}

Placebo patients received a higher amount of morphine equivalents than tapentadol patients within the first $24 \mathrm{hrs}$ after first intake of trial medication (least squares [LS] means $0.24 \mathrm{mg} / \mathrm{kg}$ [95\% CI $0.17,0.30]$ vs $0.14 \mathrm{mg} / \mathrm{kg}$ [95\% CI 0.09, 0.19]). The primary endpoint of the trial was met: the estimated LS mean treatment difference of $-0.1 \mathrm{mg} / \mathrm{kg}(95 \% \mathrm{CI}-0.18,-0.02)$ between the groups was statistically significant $(\mathrm{p}=0.0154)$ and in favor of tapentadol. The observed mean (SD) use of supplemental opioid analgesic medication (morphine equivalents) was $0.25(0.35) \mathrm{mg} / \mathrm{kg}$ bodyweight in the placebo group and $0.16(0.20) \mathrm{mg} / \mathrm{kg}$ in the tapentadol OS group. This primary endpoint result was supported by all three sensitivity analyses (Figure 3). The difference was also significantly in favor of tapentadol when the amount of opioid 


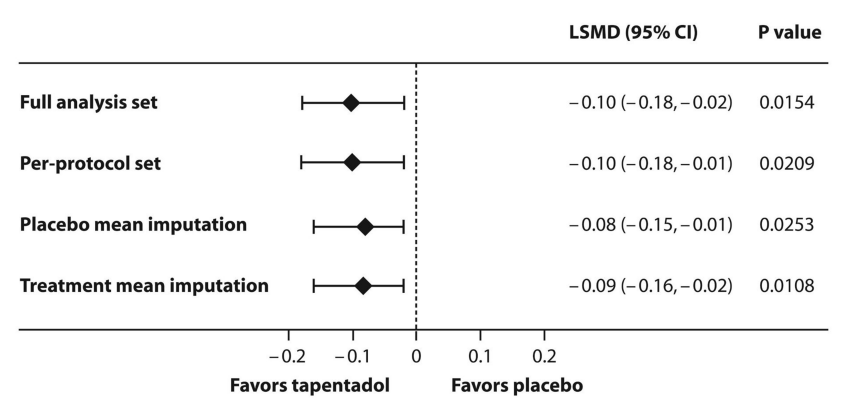

Figure 3 Least squares mean difference $(95 \% \mathrm{Cl})$ between the trial medications for the amount of supplemental opioid analgesic medication used within the first $24 \mathrm{hrs}$ after intake of first trial medication.

Abbreviation: LSMD, least squares mean difference.

analgesics taken prior to trial medication was included as a covariate in an analysis of covariance (ANCOVA) model with the same factors as the primary ANOVA model $(\mathrm{p}=0.0117)$.

\section{Secondary endpoints}

Treatment comparison for the total amount of supplemental opioid analgesic medication used within the first $12 \mathrm{hrs}$ after first dose was $0.13(95 \% \mathrm{CI} 0.09,0.17)$ for placebo and 0.08 $(95 \%$ CI $0.05,0.11)$ for tapentadol, also in favor of tapentadol with an estimated LS mean difference of -0.05 (95\% CI $-0.09,-0.0), p=0.0404$. The mean amount of supplemental opioid analgesic medication used decreased over the treatment period from 24 to $96 \mathrm{hrs;}$ it was still (numerically) smaller under tapentadol treatment up to $36 \mathrm{hrs}(0.08 \mathrm{mg} /$ $\mathrm{kg}$ [SD 0.09] vs $0.14 \mathrm{mg} / \mathrm{kg}$ [SD 0.21] for placebo) but was comparable between the treatments thereafter.

Mean pain intensity was documented prior to intake of each trial medication; Figure 4 shows the pain values for the first eight measurements. As expected in a postsurgical setting, all patients experienced some degree of pain. All had access to supplemental opioid analgesic medication and were also allowed nonopioids such as paracetamol; pain values over time thus did not differ notably between the placebo and tapentadol group, irrespective of the pain scale used.

The majority of patients found the taste of tapentadol OS acceptable and had no difficulties swallowing the medication. At the end of treatment, 71/108 (65.7\%) patients rated the taste of tapentadol OS as neutral ("a bit bad/a bit good") or better, and 82/108 (75.9\%) found swallowing "easy" or "really easy" (Figure 5). One placebo patient (1/52 [1.9\%]) and four of $108(3.7 \%)$ patients in the tapentadol group discontinued treatment because they did not like the taste of the trial medication.
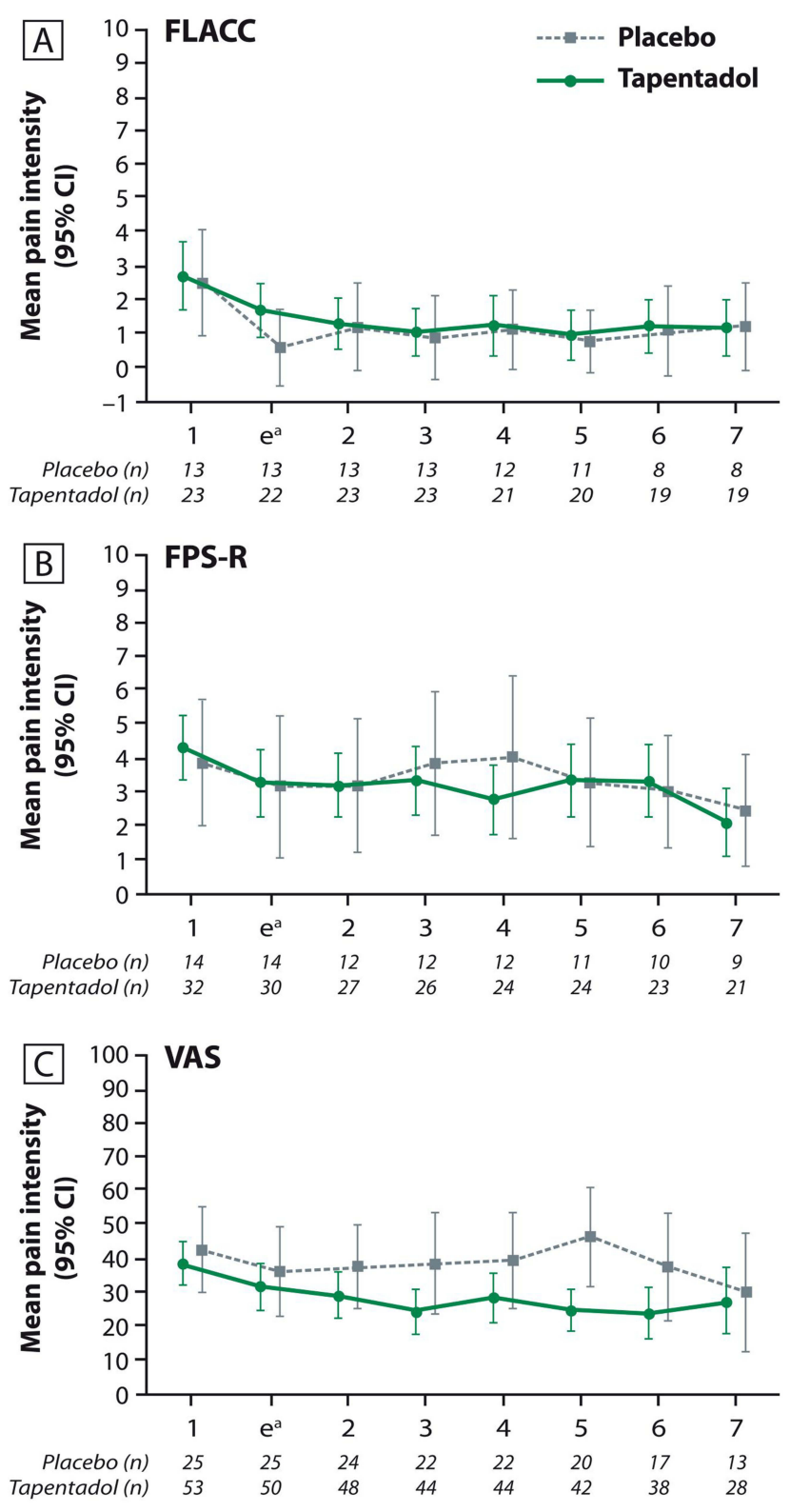

Before dose $\mathrm{x}$ of trial medication

Figure 4 Mean pain intensity ( $\pm 95 \%$ confidence interval) prior to each intake of trial medication (first seven intakes). (A) Face, Legs, Activity, Cry, and Consolability scale; (B) Faces Pain Scale-revised; (C) Visual Analog Scale.

Note: ${ }^{2}$ Assessment was performed 30-60 mins after first intake of trial medication. Abbreviations: FLACC, Face, Legs, Activity, Cry, and Consolability; FPS-R, Faces Pain Scale-revised; VAS, Visual Analog Scale.

Figure 6 shows CGIC and PGIC ratings at the end of double-blind treatment. There were no differences between placebo and tapentadol in the proportion of patients considered CGIC and PGIC responders: clinicians rated 34/52 (65.4\%) placebo patients and 73/108 $(67.6 \%)$ tapentadol patients "much" or "very much improved". Patients (or parents/guardians) also considered their condition at least much improved in $34 / 52$ 


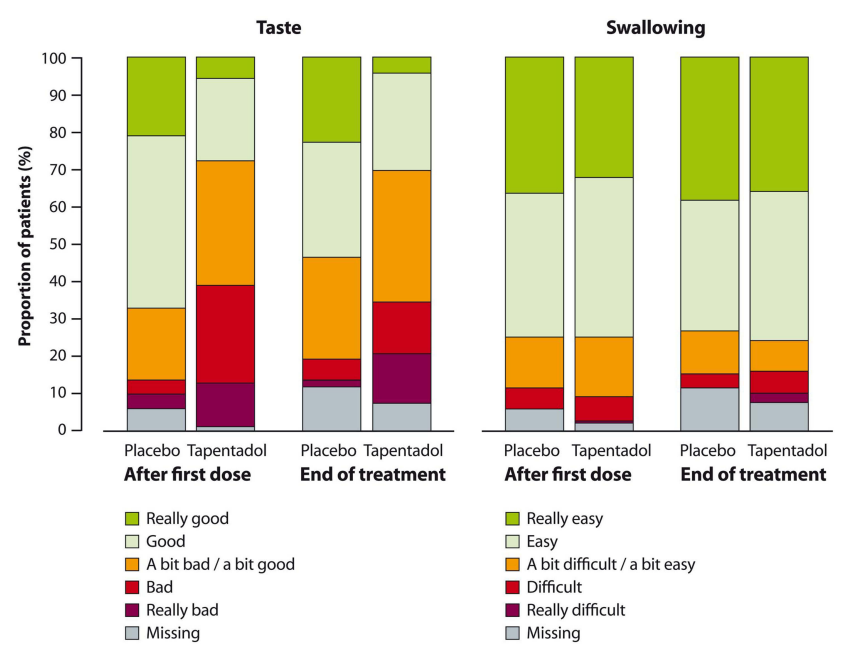

Figure 5 Palatability (taste) and acceptance (swallowing) of the trial medication after first and last doses (full analysis set).

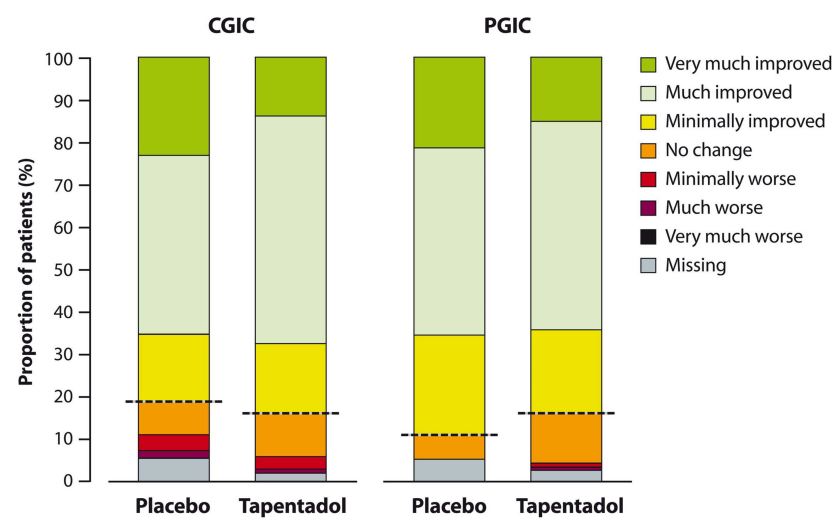

Figure 6 Clinical Global Impression of Change and Patient Global Impression of Change after completion of double-blind treatment (full analysis set).

Note: All patients above the dashed line reported improvement.

Abbreviations: CGIC, Clinical Global Impression of Change; PGIC, Patient Global Impression of Change.

(65.4\%) cases; the proportion was similar in the tapentadol group (69/108 [63.9\%]).

Four of 52 placebo patients $(7.7 \%)$ and four of 108 tapentadol patients $(3.7 \%)$ discontinued treatment due to lack of efficacy.

\section{Tolerability and safety}

Table 3 shows the TEAE profile of the trial population. Half $(26 / 52$ [50\%]) of all placebo and 62/108 (57.4\%) tapentadol patients reported overall 207 TEAEs. Incidences were higher in patients 12 to $<18$ years than in the two younger age groups. However, the number of patients available for age group specific analyses was lower in the 2 younger age groups which needs to be considered when looking at the differences in the observed
TEAE rates by age group. The most common TEAEs were vomiting, nausea, and constipation in both treatment groups. Pyrexia was documented for $10 / 108$ patients in the tapentadol group (9.3\%) compared to $1 / 52$ placebo patients $(1.9 \%)$, mostly in the older age group $(n=7)$; none were considered by the investigator to be related to treatment. The majority of TEAEs (138/207 [66.7\%]) started within $24 \mathrm{hrs}$ after first dose of trial medication. Nearly all (205/207 [99\%]) were mild or moderate in intensity; two severe TEAEs occurred under tapentadol (abdominal distension which was unlikely and headache which was probably/likely related to the trial medication according to the investigator's assessment; both led to discontinuation of trial medication). Two of 108 tapentadol patients $(1.9 \%)$ experienced serious adverse events, both considered unlikely related to the trial medication by the investigator: one male adolescent with a history of Crohn's disease had an abdominal abscess of moderate intensity, one male child in the 6 to $<12$ years age group experienced a seizure of moderate intensity, likely due to hyponatremia secondary to cerebral salt wasting in the investigator's judgment. Forty of all 160 patients experienced TEAEs related to the trial medication, most of those known adverse reactions to tapentadol: 11/52 (21.2\%) patients in the placebo and 29/108 (26.9\%) patients in the tapentadol group. Treatment was discontinued due to a TEAE in $12 /$ 160 patients $(7.5 \%)$ overall; $2 / 52$ patients $(3.8 \%)$ under placebo and $10 / 108$ patients $(9.3 \%)$ under tapentadol treatment. Nine of these patients were in the 12 to $<18$ years age group. There was no difference in the time to treatment discontinuation due to a TEAE between the treatment arms in the first $24 \mathrm{hrs}$ of treatment.

Clinical chemistry and hematology values were mostly similar at baseline and end of treatment in both treatment arms. Most of the laboratory findings were attributed by the investigators to the surgery and postsurgical recovery. There were no clinically relevant changes in ECG, pulse rate, or blood pressure. Low respiratory rates occurred more often in tapentadol patients $(27 / 108$ [25\%] vs $9 / 52$ [17.3\%] for placebo) at the end of treatment. However, in both treatment arms the mean respiratory rates at the end of treatment were similar to those at baseline (tapentadol: 19.3 vs 19.5 breaths per minute [bpm]; placebo: 21.5 vs $19.9 \mathrm{bpm}$ ). The same was true for mean oxygen saturation values (tapentadol: 97.9 vs 98.0\%; placebo: 98.1 vs 98.1\%). Oxygen saturation decrease, hypoxia, or $\mathrm{PO}_{2}$ decrease were reported as TEAEs in one placebo and seven tapentadol patients. Most of the events were mild, 


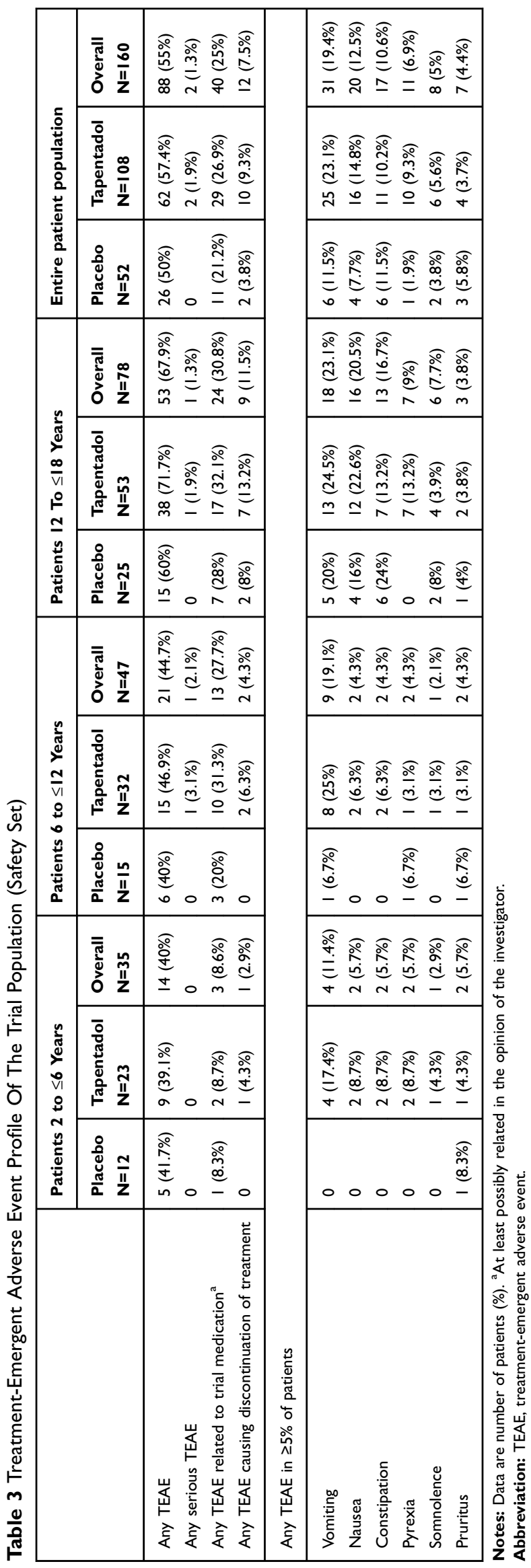

not or unlikely related to the trial medication and resolved quickly. Furthermore, confounding factors were identified for most of the events. Only two TEAEs of hypoxia were considered possibly related to tapentadol (one with mild, one with moderate intensity). The first event occurred in a patient who underwent a mitral valve replacement; the rate of oxygen flow to the patient was increased from $1 \mathrm{~L} / \mathrm{min}$ to $2 \mathrm{~L} / \mathrm{min}$ and the amount of supplemental opioid analgesic medication per NCA/PCA push was decreased from $0.1 \mathrm{mg}$ to $0.08 \mathrm{mg}$. The second event occurred in a patient who underwent thoracic surgery for pectus carinatum; the patient was placed on incentive spirometry. There were no cases of suicidal ideation or behavior at the end of treatment. Sedation of moderate intensity was reported as a TEAE for two patients and was considered possibly related to tapentadol in both cases. One of these patients was discontinued from tapentadol; for both patients, recovery was reported. Slightly more tapentadol patients were moderately or deeply sedated but all could be aroused. Most patients were awake and alert or minimally sedated before the next administration of trial medication.

\section{Discussion}

This clinical trial was designed to determine the efficacy and safety/tolerability of multiple doses of tapentadol OS $(1.25 \mathrm{mg} / \mathrm{kg})$ in the treatment of moderate to severe acute pain in children and adolescents aged 2 to $<18$ years using postsurgical pain as a model.

Pediatric trials should aim to provide maximum information using a minimum number of patients; efficacy trials, however, need adequate power to determine efficacy and sufficient patient numbers to provide a robust safety database. ${ }^{1}$ Achieving an adequate sample size can be difficult. Nearly one-fifth (19\%) of all randomized clinical pediatric trials registered on the ClinicalTrials.org website between 2008 and 2010 were discontinued early with poor recruitment and trial conduct problems being the main issues. ${ }^{32}$ To that end, we aimed at accommodating the requirements of trial sites and their preferred surgical interventions and local postsurgical standards of care as much as possible. Surgical procedures were not to affect the absorption or safety profile of tapentadol but had to require opioid pain therapy over a sufficient time interval in order to measure the primary endpoint of the trial. Inclusion/exclusion criteria were set flexible enough to include a variety of surgeries, thereby allowing recruitment of all targeted age groups. Further operational challenges concerned the multiple dosing regimen and the 
duration of treatment. Dosing intervals had to be sufficiently flexible to avoid interference with sleep but at the same time had to be strict enough to allow for sufficient exposure to tapentadol. This was achieved by extending the normal 4-hr dosing interval to a maximum of $6 \mathrm{hrs}$ in case a patient was asleep. The time frame for measuring the primary efficacy endpoint (i.e., the minimum treatment duration to consider a patient as a treatment completer) was chosen as short as possible to take into account the reduced need for analgesia over time. At the same time, the protocol allowed for prolonged treatment in case further treatment was considered beneficial by the physician. This flexibility reaped the advantage of obtaining further valuable safety and efficacy data, thereby achieving the objectives of this trial.

Tapentadol was more efficacious (statistically significant) than placebo as shown by the comparison of the amount of supplemental opioid analgesic medication used within the first $24 \mathrm{hrs}$ after first dose of trial medication between tapentadol and placebo. The result was confirmed by sensitivity analyses and supported by the secondary efficacy analyses. The majority of the children had no difficulty swallowing tapentadol OS; palatability and acceptability of the medication were considered sufficient to ensure intake compliance for all age groups investigated.

The patient population recruited for this trial reflects the general pediatric population in the postsurgical setting covering a wide age range from 2 to $<18$ years, a wide variety of surgeries, and different modes of supplemental opioid analgesic medication administration (i.e., PCA and NCA) and thus permits concluding the efficacy and safety of tapentadol across a wide range of postsurgical pain conditions. The comparable PGIC and CGIC responder results for tapentadol and placebo patients indicate that treatments (placebo/tapentadol plus supplemental analgesia) were assessed as equally beneficial and that the chosen trial design proved ethical and adequate to investigate the efficacy of tapentadol in this pediatric population. Owing to the trial design, allowing for immediate use of rescue pain medication, all patients had constant access to supplemental opioid analgesic medication. The use of other nonopioid analgesics was also permitted in accordance with local standard of care. Therefore, pain values were not expected to differ between the treatment groups over time. Efficacy was thus established by comparing the amount of supplemental opioid analgesic medication used in the tapentadol arm with that used in the placebo arm (opioid sparing).

The clinical relevance of the efficacy results in this trial is challenging to assess given that the efficacy endpoints deviate from those more commonly used. The effect of tapentadol OS on supplemental opioid use (treatment group difference of $0.1 \mathrm{mg} / \mathrm{kg}$ body weight of morphine equivalents in $24 \mathrm{hrs}$ comparing placebo to tapentadol) is less pronounced than published for other nonopioid compounds tested using the same design. ${ }^{30,31}$ In contrast to these trials where supplemental opioid analgesic medication was administered solely using PCA and the focus was on postsurgical pain following pectus correction surgery in 10-15-year-old patients ${ }^{30}$ and spinal fusion surgery in 918-year-old patients, ${ }^{31}$ the present trial included a broader age range ( 2 to $<18$ years), a wide variety of different surgery types, and administration of supplemental opioid analgesic medication by PCA or NCA. This may explain the observed difference between initial assumptions and trial outcome.

In adult chronic pain trials, reductions in pain intensities $\geq 30 \%$ and $\geq 50 \%$ are considered to reflect at least moderate clinically important changes and substantial improvements, respectively. ${ }^{33}$ In analogy, a reduction of $30-50 \%$ in the use of supplemental opioid analgesic medication (primary efficacy endpoint) might be considered to be clinically relevant. The LS mean treatment difference between placebo and tapentadol OS was $0.1 \mathrm{mg} / \mathrm{kg}$ body weight, i.e., a $41.7 \%$ lower supplemental opioid analgesic medication use in the tapentadol group, thus may be considered a clinically meaningful change. Following treatment with trial medication supplemental opioid analgesic medication use decreased by $44 \%$ under placebo compared to $72 \%$ under tapentadol. This reduction is considerably more pronounced ( $28 \%$ difference) in the tapentadol arm supporting clinical relevance of the observed treatment difference. Furthermore, the combined mean total amount of supplemental opioid analgesic medication was $0.55 \mathrm{mg} /$ $\mathrm{kg}$ morphine equivalents during the $24 \mathrm{hrs}$ before treatment initiation. Based on this number also from the perspective of the total amount of supplemental opioid analgesic medication "saved" in the tapentadol group, i.e., $0.1 \mathrm{mg} / \mathrm{kg}$ body weight, a clinically relevant change may be concluded.

The tolerability profile of tapentadol OS was as expected for a centrally acting analgesic in the postsurgical setting. The observed TEAEs were generally in line with the known safety profile of tapentadol. This trial thus adds 
to the body of evidence of safety and tolerability observed in adult patients. Incidences of TEAEs were higher for the group of adolescents (12 to $<18$ years) than for younger patients which might be due to the fact that older children are generally better able to verbally express the occurrence of side effects. In addition, the higher amount of supplemental opioid analgesic medication used in the older group might have had an impact. The most frequent AEs vomiting $(23.1 \%$ vs $11.5 \%$ for placebo) and nausea ( $14.8 \%$ vs $7.7 \%$ ) occurred more often with tapentadol. The fact that the majority of tapentadol patients received two medications with an opioid mechanism of action (tapentadol and morphine/hydromorphone) could be a likely explanation for this observation.

The incidence of pyrexia was higher with tapentadol ( $9.3 \%$ vs $1.9 \%$ of patients). Pyrexia is not a known AE of tapentadol treatment, and none of the events were considered by the investigator to be related to tapentadol. Confounding factors which might explain the higher incidence compared to placebo included inflammation reactions due to surgery or an orthopedic implant, pre-existing fever, perisplenitis, skin inflammation, or fever due to blood transfusions. It should be noted that the tolerability profile might have been influenced by the postsurgical state and administration of morphine/hydromorphone as supplemental analgesia.

Respiratory depression is a known complication of opioid administration. ${ }^{34}$ Under tapentadol, only a few incidences suggestive of respiratory depression have been documented in adult trials in the postsurgical setting. ${ }^{14}$ In the present trial, patients were in the postsurgical state and were receiving multiple medications including morphine/hydromorphone in addition to the trial medication. Consequently, AEs due to the underlying condition and opioid treatment were expected, so vital signs, sedation scores, and oxygen saturation were carefully monitored throughout the trial. Respiratory depression was not documented as an AE. The incidence of AEs suggestive of respiratory depression under tapentadol was low; of the seven cases, only mild and moderate hypoxia were considered possibly related in two tapentadol patients who were at higher baseline risk due to the type of surgery performed. There was no relevant difference to placebo in mean oxygen saturation or respiratory rate. The majority of the patients were awake and alert or minimally sedated before the next administration of trial medication. Patients who were moderately or deeply sedated could all be aroused. Overall, these findings indicate that multiple doses of tapentadol OS were generally well tolerated in this pediatric population and that no higher susceptibility to gastrointestinal or CNS reactions are expected compared to adults.

Based on data obtained from the complete pediatric development program, tapentadol OS was recently approved in the EU in children 2 to $<18$ years of age for the treatment of moderate to severe acute pain which can be adequately managed only with opioid analgesics. As pediatric clinical investigations have so far only been carried out in the hospital setting, the currently labelled use is restricted to the hospital setting where appropriate equipment to enable respiratory support is available. The recommended approved single dose of tapentadol OS is $1.25 \mathrm{mg} / \mathrm{kg}$ body weight every 4 hrs; duration of treatment should not exceed 3 days as data for longer treatment periods are not yet available. ${ }^{35}$

\section{Conclusions}

The data from this trial confirm that tapentadol OS can be effectively used in children ( 2 years and older) for the treatment of moderate to severe acute pain. Tapentadol OS was generally well tolerated and no new adverse drug reaction or changed severity of a known adverse reaction was identified compared to the tapentadol safety profile in adults. It further supports the positive benefit-risk ratio of tapentadol for all age groups from 2-year-old children and adults. The palatability and acceptability of the medication was considered sufficient to ensure intake compliance in all age groups. This trial provides robust clinical trial evidence that tapentadol OS can be effectively used to treat moderate to severe acute pain in children, in contrast to other analgesics for which use is based on empirical evidence and clinical experience. It also provides an alternative treatment in older pediatric populations for whom approved drugs are not suitable either due to the administrative route, e.g., PCA or efficacy and/or tolerability issues.

\section{Abbreviations}

AE, adverse event; ANCOVA, analysis of covariance; ANOVA, analysis of variance; CGIC, Clinical Global Impression of Change; ECG, electrocardiogram; FAS, full analysis set; FLACC, Face, Legs, Activity, Cry, and Consolability scale; FPS-R, Faces Pain Scale-revised; LS, least squares; MOR, $\mu$-opioid receptor agonism; NCA, nurse-controlled analgesia; NRI, noradrenaline reuptake inhibition; OS, oral solution; PCA, patient-controlled analgesia; PGIC, Patient Global Impression of Change; PK, pharmacokinetic;TEAE, treatment-emergent adverse event; VAS, Visual Analog Scale. 


\section{Data sharing statement}

The authors will share upon request the final clinical trial report. They will also consider on a case-by-case basis requests for access to other documents and/or data including individual patient data. There is no limitation regarding document/data availability.

\section{Acknowledgments}

The authors thank all patients, parents/legal guardians, investigators, and the trial site teams involved in this investigation. The trial was funded by Grünenthal $\mathrm{GmbH}$. Writing assistance was provided by Elke Grosselindemann and Birgit Brett and was funded by Grünenthal GmbH.

\section{Disclosure}

Christoph Beuter, Gisela Volkers, Tatjana Radic, and Jutta Goldberg are employees of Grünenthal GmbH. John van den Anker is a paid consultant for Grünenthal. The authors report no other conflicts of interest in this work.

\section{References}

1. Eerdekens M, Beuter C, Lefeber C, van den Anker J. The challenge of developing pain medications for children: therapeutic needs and future perspectives. J Pain Res. 2019; 12:1649-1664.

2. Regulation (EC) No 1901/2006 of the European Parliament and of the Council of 12 December 2006 on medicinal products for paediatric use, OJ L 378, 27.12.2006. Available from: https://ec.europa.eu/health/ sites/health/files/files/eudralex/vol-1/reg_2006_1901/reg_2006_1901 en.pdf. Accessed September 28, 2018.

3. Food and Drug Administration. Regulations requiring manufacturers to assess the safety and effectiveness of new drugs and biological products in pediatric patients-FDA. Final rule. Fed Regist. 1998;63(231):6663166672

4. American Academy of Pediatrics, American Pain Society. The assessment and management of acute pain in infants, children, and adolescents. Pediatrics. 2001;108(3):793-797. doi:10.1542/peds. 108.3.793

5. Association of Paediatric Anaesthetists of Great Britain and Ireland. Good practice in postoperative and procedural pain management, 2nd edition. Paediatr Anaesth. 2012;22(suppl1):1-79. doi:10.1111/j.14609592.2012.03838.x

6. Tzschentke TM, De Vry J, Terlinden R, et al. Tapentadol hydrochloride. Analgesic, mu-opioid receptor agonist, noradrenaline reuptake inhibitor. Drugs Future. 2006;31:1053-1061.

7. Hale M, Upmalis D, Okamoto A, Lange C, Rauschkolb C. Tolerability of tapentadol immediate release in patients with lower back pain or osteoarthritis of the hip or knee over 90 days: a randomized, doubleblind study. Curr Med Res Opin. 2009;25:1095-1104. doi:10.1185/ 03007990902816970

8. Hartrick C, Van Hove I, Stegmann JU, Oh C, Upmalis D. Efficacy and tolerability of tapentadol immediate release and oxycodone HCL immediate release in patients awaiting primary joint replacement surgery for end-stage joint disease: a 10-day, phase III, randomized, double-blind, active- and placebo-controlled study. Clin Ther. 2009;31:260-271. doi:10.1016/j.clinthera.2009. 02.009
9. Daniels SE, Upmalis D, Okamoto A, Lange C, Haeussler J. A randomized, double-blind, phase III study comparing multiple doses of tapentadol IR, oxycodone IR, and placebo for postoperative (bunionectomy) pain. Curr Med Res Opin. 2009;25:765-776. doi:10.1185/03007990902728183

10. Daniels S, Casson E, Stegmann JU, et al. A randomized, doubleblind, placebo-controlled phase 3 study of the relative efficacy and tolerability of tapentadol IR and oxycodone IR for acute pain. Curr Med Res Opin. 2009;25:1551-1561. doi:10.1185/0300799090295 2825

11. Vorsanger GJ, Klopfer AM, Xiang J, Benson CJ, Moskovitz BL, Rosenthal NR. Immediate-release tapentadol or oxycodone for treatment of acute postoperative pain after elective arthroscopic shoulder surgery: a randomized, phase IIIb study. J Opioid Manage. 2013;9:281-290. doi:10.5055/jom.2013.0170

12. Biondi D, Xiang J, Benson C, Etropolski M, Moskovitz B, Rauschkolb C. Tapentadol immediate release versus oxycodone immediate release for treatment of acute low back pain. Pain Physician. 2013;16:E237-E246.

13. Vorsanger GJ, Farrell J, Xiang J, Chow W, Moskovitz BL, Rosenthal NR. Tapentadol, oxycodone or placebo for acute pain of vertebral compression fractures: a randomized phase IIIb study. Pain Manage. 2013;3:109-118. doi:10.2217/pmt.13.5

14. Viscusi E, Allard R, Sohns M, Eerdekens M. Tapentadol immediate release for moderate to severe acute post-surgery pain. $J$ Opioid Manage. 2019;15:51-67. doi:10.5055/jom.2019.0486

15. Baron R, Eberhart L, Kern KU, et al. Tapentadol prolonged release for chronic pain: a review of clinical trials and 5 years of routine clinical practice data. Pain Pract. 2017;17:678-700. doi:10.1111/ papr. 12515

16. Raffa RB, Elling C, Tzschentke TM. Does 'strong analgesic' equal 'strong opioid'? Tapentadol and the concept of ' $\mu$-load'. Adv Ther. 2018;35:1471-1484. doi:10.1007/s12325-018-0778-x

17. Electronic Medicines Compendium. Palexia $50 \mathrm{mg}$ film-coated tablets. Available from: https://www.medicines.org.uk/emc/medi cine/28375. Accessed October 15, 2018.

18. Smit JW, Oh C, Rengelshausen J, et al. Effects of acetaminophen, naproxen, and acetylsalicylic acid on tapentadol pharmacokinetics: results of two randomized, open-label, crossover, drug-drug interaction studies. Pharmacotherapy. 2010;30(1):25-34. doi:10.1592/ phco.30.1.25

19. Finkel J, Goldberg J, Rosenburg R, et al. First evaluation of tapentadol oral solution for the treatment of moderate to severe acute pain in children aged 6 to $<18$. J Pain Res. 2019;12:1925-1936. doi:10.2147/ JPR.S197348

20. Muse D, Tarau E, Lefeber C, et al. Pharmacokinetics, safety, and efficacy of tapentadol oral solution for treating moderate to severe pain in pediatric patients. J Pain Res. 2019;12:1777-1790.

21. Watson E, Khandelwal A, Freijer J, van den Anker J, Lefeber C, Eerdekens M. Population pharmacokinetic modeling to facilitate dose selection of tapentadol in the pediatric population. $J$ Pain Res. 2019;12:2835-2850.

22. Berde CB, Walco GA, Krane EJ, et al. Pediatric analgesic clinical trial designs, measures, and extrapolation: report of an FDA scientific workshop. Pediatrics. 2012;129(2):354-364. doi:10.1542/peds.20103591

23. Walco GA, Kopecky EA, Weisman SJ, et al. Clinical trial designs and models for analgesic medications for acute pain in neonates, infants, toddlers, children, and adolescents: ACTTION recommendations. Pain. 2018;159:193-205. doi:10.1097/j.pain.0000000000001104

24. Kossowsky J, Donado C, Berde CB. Immediate rescue designs in pediatric analgesic trials: a systematic review and meta analysis. Anesthesiology. 2015;122(1):150-171. doi:10.1097/ALN.0000000000000445

25. Merkel SI, Voepel-Lewis T, Shayevitz JR, Malviya S. The FLACC: a behavioral scale for scoring postoperative pain in young children. Pediatr Nurs. 1997;23:293-297. 
26. Hicks CL, von Baeyer CL, Spafford PA, van Korlaar I, Goodenough B. The faces pain scale-revised: toward a common metric in pediatric pain measurement. Pain. 2001;93:173-183. doi:10.1016/s0304-3959 (01)00314-1

27. Guy W. ECDEU assessment manual for psychopharmacology. (DHEW Publication no. ADM 76-338). Washington, DC: US Government Printing Office; 1976.

28. Guinard JX. Sensory and consumer testing with children. Trends Food Sci Technology. 2001;11(8):273-283. doi:10.1016/S0924-2244 (01)00015-2

29. Posner K, Brent D, Lucas C, et al. Columbia-Suicide Severity Rating Scale (C-SSRS). Available from: http://cssrs.columbia.edu/wp-content/uploads/ C-SSRS Pediatric-SLC 11.14.16.pdf. Accessed October 22, 2018.

30. Rugyte D, Kokki H. Intravenous ketoprofen as an adjunct to patientcontrolled analgesia morphine in adolescents with thoracic surgery: a placebo controlled double-blinded study. Eur J Pain. 2007;11:694699. doi:10.1016/j.ejpain.2006.11.001
31. Rusy LM, Hainsworth KR, Nelson TJ, et al. Gabapentin use in pediatric spinal fusion patients: a randomized, double-blind, controlled trial. Anesth Analg. 2010;110:1393-1398. doi:10.1213/ANE. 0b013e3181d41dc2

32. Pica N, Bourgeois F. Discontinuation and nonpublication of randomized clinical trials conducted in children. Pediatrics. 2016;138(3): e20160223. doi:10.1542/peds.2016-0223

33. Dworkin RH, Turk DC, Wyrwich KW, et al. Interpreting the clinical importance of treatment outcomes in chronic pain clinical trials: IMMPACT recommendations. J Pain. 2008;9(2):105-121. doi:10.10 16/j.jpain.2007.09.005

34. Dahan A, Aarts L, Smith TW. Incidence, reversal, and prevention of opioid-induced respiratory depression. Anesthesiology. 2010; 112:226-238. doi:10.1097/ALN.0b013e3181c38c25

35. Electronic Medicines Compendium. Palexia oral solution $20 \mathrm{mg} / \mathrm{mL}$. Available from: https://www.medicines.org.uk/emc/product/5346/ smpc. Accessed November 19, 2018.

\section{Publish your work in this journal}

The Journal of Pain Research is an international, peer reviewed, open access, online journal that welcomes laboratory and clinical findings in the fields of pain research and the prevention and management of pain. Original research, reviews, symposium reports, hypothesis formation and commentaries are all considered for publication. The manuscript management system is completely online and includes a very quick and fair peer-review system, which is all easy to use. Visit http:// www.dovepress.com/testimonials.php to read real quotes from published authors. 